\title{
Preparation of Islets of Langerhans from the Hamster Pancreas
}

\author{
Lawrence Rosenberg, M.D., Ph.D., ${ }^{1}$ Richard Schwartz, M.D., \\ Donald C. Dafoe, M.D., Susan Clarke, B.S., Jeremiah G. TurcotTe, M.D., \\ AND AARON I. VINIK, M.D. \\ Departments of Surgery and Internal Medicine, Division of Endocrinology and Metabolism, \\ University of Michigan Medical Center, Ann Arbor, Michigan
}

Submitted for publication January 26, 1987

\begin{abstract}
We describe a method for the preparation of viable islets from the pancreas of normal 8-week-old female Syrian golden hamster, based on the injection of the pancreatic duct with collagenase and on mechanical dissociation which liberates islets that maintain their normal morphological appearance and physiologic function. In a series of 11 animals, we examined the dose response of intraductal collagenase on islet yield. The mean number of isolated islets was 423 with a range from 130 to 873 per pancreas. Islet yield was most dependent on the concentration of collagenase solution used to inject the duct. The optimal concentration was determined to be $3.5 \mathrm{mg} / \mathrm{ml}$ when a total volume of $3.0 \mathrm{ml}$ was injected. Islets responded to glucose stimulus in a normal biphasic pattern. Mesh filtration, rather than Ficoll, can be performed rapidly and results in a high yield of functional islets with minimal contamination by acinar tissue. 1988 Academic Press, Inc
\end{abstract}

\section{INTRODUCTION}

The isolation of islets of Langerhans is a difficult problem because islets comprise only about $10 \%$ of the pancreas. The size, architecture, and compact fibrous nature of the human pancreas, in particular, present additional problems for the islet separation procedure [1]. The isolation of islets is of interest because islet transplantation holds great potential for the treatment of diabetes mellitus [1] and because islets have been implicated in the early stage of experimental pancreatic carcinogenesis [2].

Currently employed techniques for the separation of islets from surrounding exocrine tissue are related to a method originally developed in the rat pancreas by Moskalewski [3] and subsequently improved upon by Lacy and Kostianovsky [4]. The essential features of this technique are the initial distension of the pancreas by the ductal administration of normal saline, mincing of the pancreas into small pieces, incubation of the

\footnotetext{
'To whom reprint requests should be addressed at Department of Surgery, Montreal General Hospital, 1650 Cedar Avenue, Livington Hall \#9860, Montreal, Quebec H3G 1 A4.
}

tissue in collagenase with concomitant mechanical agitation, and centrifugation of the dispersed tissue on a Ficoll density gradient. When similar techniques have been employed to isolate murine islets, the overall yield has been very low $[5,6]$. Yields of $100-200$ islets per hamster pancreas have been reported utilizing this method, with handpicking of islets used in the final stage of separation [7]. While this technique results in a relatively pure islet preparation, it is time-consuming and the yield is low. More recently high yields of islets have been obtained from canine and human pancreases when collagenase was initially injected into the duct to distend the parenchyma $[8,9]$.

We describe here an adaptation of the latter method to the pancreas of the Syrian golden hamster that increased the yield of islets that function normally in response to glucose.

\section{MATERIALS AND METHODS}

\section{Animals}

Eleven outbred female Syrian golden hamsters (85-95 $\mathrm{g}$ body weight), 8 weeks of age (CBL, Syracuse, NY), were used in this 
study. They were housed in plastic cages and fed RMH 1000 (Charles River, Syracuse, NY) and water ad libitum.

Islet Isolation

\section{Preparation of Media}

Washing medium. Hanks' balanced salt solution (HBSS) (GIBCO Laboratories, Grand Island, NY) was used as a washing medium. The $\mathrm{pH}$ was adjusted to 7.4 using sterile sodium bicarbonate and was then kept on ice for the duration of the extraction procedure.

Digestion medium. Collagenase Type V-S (Sigma Chemical Co., St. Louis, MO) was reconstituted in HBSS to a final concentration of $3.5 \mathrm{mg} / \mathrm{ml}$. Three milliliters of the digestion medium was then drawn up in a sterile $5-\mathrm{ml}$ syringe which was then connected to a 30-gauge needle fitted to a 3-in. length of polyethylene tubing (Clay Adams, New York, NY). The filled syringe was then kept on ice until required. The unused portion of the digestion medium was immediately frozen at $-40^{\circ} \mathrm{C}$.

Culture medium. RPMI 1640 (GIBCO Laboratories, NY) was adjusted with sterile sodium bicarbonate to a $\mathrm{pH}$ of 7.4. Next fetal bovine scrum (GIBCO Laboratories, NY) was heat inactivated at $56^{\circ} \mathrm{C}$ for $30 \mathrm{~min}$. The culture medium was prepared by combining RPMI $1640(89 \mathrm{ml})$, fetal bovine serum (10 $\mathrm{ml}$ ), and penicillin/streptomycin solution (1 $\mathrm{ml}$ ) (GIBCO Laboratories, NY). This was refrigerated until required.

\section{Removal of the Pancreas}

Each animal was anesthetized using $0.35-0.40 \mathrm{ml}$ of phenobarbital $(20 \mathrm{mg} / \mathrm{ml})$ administered intraperitoneally using a 21 gauge needle. The abdominal region was then washed with a $50 \%$ Betadine solution. The abdomen was opened using a long midline incision and a small mastoid retractor was placed to expose the abdominal viscera. The lobes of the liver were retracted craniad to expose the common hepatic and bile ducts. A Q-tip was employed to retract the duodenal loop caudad.
Utilizing a dissecting microscope with appropriate lighting, a 5-O silk suture was placed around the common bile duct at its entrance into the duodenum. To prevent the development of intraparenchymal hemorrage when the digestion medium was injected into the ductal system of the pancreas the chest cavity was entered by dividing the diaphragm and sternum, and the aorta and inferior vena cava were clamped with a micro bulldog clamp. Three milliliters of the digestion medium (enough to cause the gland to swell) was then slowly injected via a polyethylene catheter placed into the common bile duct proximal to the duodenum causing the pancreatic tissue to swell. The pancreas was then resected and placed on a $60 \times 15-\mathrm{mm}$ sterile petri dish containing chilled washing medium. Fatty tissue and adherent lymph nodes were removed by sharp dissection. The covered dish was then transferred to a water bath at $37^{\circ} \mathrm{C}$ for 40 min to allow for collagenase digestion of the tissue.

\section{Islet Separation}

The incubation dish was removed from the water bath, the excess washing medium was pipetted off, and the pancreas digest was decanted into a $15-\mathrm{ml}$ conical centrifuge tube into which was added $10 \mathrm{ml}$ of cold HBSS in order to halt the digestion process. The tissue was then mechanically dissociated by vortexing and the resulting tissue suspension was centrifuged at $1400 \mathrm{rpm}$ for $15 \mathrm{sec}$. The supernatant was discarded and the tissue pellet was resuspended in $10 \mathrm{ml}$ of cold HBSS. This suspension was then washed three more times.

Following the last wash, the tissue suspension was pipetted onto a sterile $840-\mu \mathrm{m}$ stcel mesh filter (Bellco Glass, Inc., Vineland, NJ) that had been taped to the mouth of a sterile glass funnel. The filter was then washed with cold HBSS and the filtrate, containing islets and acini, was collected in a 50-ml conical centrifuge tube. This filtrate was pipetted onto a $140-\mu \mathrm{m}$ steel mesh filter which was then washed with cold HBSS. The filtrate, 
which contained small islets and intact acini, was collected in a sterile $50-\mathrm{ml}$ centrifuge tube. The filter was then inverted and washed with cold HBSS so that the tissue on the filter, which consisted primarily of large islets, was collected in another $50-\mathrm{ml}$ conical centrifuge tube. The filtrate containing the small islets and acini was then pipetted onto a $94-\mu \mathrm{m}$ steel mesh which was washed with cold HBSS. The filtrate, which now contained acinar debris, was discarded. The 94- $\mu \mathrm{m}$ filter was then inverted and washed with cold HBSS and the small islets which were trapped on the mesh were collected in a centrifuge tube.

The centrifuge tubes containing the large (greater than $140-\mu \mathrm{m}$ diameter) and smaller (less than $140-\mu \mathrm{m}$ diameter) islets were next centrifuged at $1300 \mathrm{rpm}$ for $45 \mathrm{sec}$. The supernatants were then aspirated and the islet pellets were resuspended in $7-10 \mathrm{ml}$ of culture medium. The contents of the centrifuge tubes were then divided among four petri dishes $(100 \times 20 \mathrm{~mm})$.

\section{Microscopy}

Following the isolation procedure, islet morphology was examined by dark-field illumination under a Nikon diaphot inverted microscope (Model No. 108) (Nikon, Garden City, NJ). The occasional large pieces of acinar debris were removed by transfer pipet under microscopic guidance. Islet diameter was measured using an eyepiece reticle.

\section{Islet Culture}

The islets which were isolated by the mesh filtration technique were then incubated overnight in a humidified atmosphere of $95 \%$ air: $5 \%$ carbon dioxide at $37^{\circ} \mathrm{C}$.

\section{In Vitro Perifusion of Islets}

We examined insulin secretion using the perifusion system described by Lacy and colleagues [10]. Briefly it is as follows. The perifusion apparatus consisted of a doubleheaded peristaltic pump (Harvard Apparatus Co., Millis, MA), polyethylene tubing (inner diameter of $\frac{1}{16}$ in.), a water bath, and a plastic perifusion chamber (Millipore, Bedford, MA) containing a 5- $\mu \mathrm{m}$ Millipore filter (Gelman). The perifusion chamber was maintained at $37^{\circ} \mathrm{C}$ in a water bath and constantly gassed, throughout the period of perifusion with a defined medium gassed with $5 \%$ $\mathrm{CO}_{2}: 95 \% \mathrm{O}_{2}$ [10]. The pump ensures the delivery of the medium from the reservoir in the water bath to the perifusion chamber at a flow rate of $0.9-1.0 \mathrm{ml} / \mathrm{min}$. The chamber was maintained at $37^{\circ} \mathrm{C}$ by clamping it beneath the surface of the water bath. The perifusate was collected in $10-\mathrm{ml}$ glass tubes at 1 -min intervals using an automated fraction collector.

One hundred handpicked islets were then transferred to a small cup containing perifusion medium (glucose concentration of 0.3 $\mathrm{mg} / \mathrm{ml}$ ) and were then pipetted onto the surface of a Millipore filter. Islets were perifused for $35 \mathrm{~min}$ with a low glucose $(0.3 \mathrm{mg} / \mathrm{ml})$ solution. After this time, a high-glucose-containing perifusion medium was substituted $(3.0 \mathrm{mg} / \mathrm{ml})$, and perifusion was continued for $45 \mathrm{~min}$. Samples were taken for insulin assay at $1-\mathrm{min}$ intervals.

\section{Determination of Insulin Secretion by Islets in Vitro}

The insulin content of each aliquot of perifusate was determined by a specific insulin radioimmunoassay [11]. Hamster insulin cross-reacts with the porcine trace used in this assay. Human insulin was used as the standard. The rate of insulin secretion (mean \pm SEM) is expressed as microunits of insulin per islet per minute.

\section{Statistical Analysis}

$\chi^{2}$ analysis was applied to a contingency table to test the independence of collagenase concentration and islet yield. A probability value $(P)$ of $<0.01$ was considered significant.

\section{RESULTS}

\section{Isolation of Pancreatic Islets}

Large numbers of islets of Langerhans were readily isolated from the pancreas of the 
TABLE 1

THE NUMBER OF ISLETS ISOLATED BY DIFFERENTIAL MESH FILTRATION

\begin{tabular}{ccc}
\hline Animal & $\begin{array}{c}\text { Collagenase } \\
(\mathrm{mg} / \mathrm{ml})\end{array}$ & Islet number \\
\hline 1 & 5.0 & 261 \\
2 & 5.0 & $232^{a}$ \\
3 & 4.0 & 330 \\
4 & 4.0 & 360 \\
5 & 3.5 & 747 \\
6 & 3.5 & $404^{a}$ \\
7 & 3.5 & 728 \\
8 & 3.5 & 873 \\
9 & 3.5 & $434^{a}$ \\
10 & 2.0 & 130 \\
11 & 2.0 & 150 \\
\hline
\end{tabular}

${ }^{a}$ Intra parenchymal injection of collagenase solution.

Syrian golden hamster using the differential steel mesh filtration method. In the 11 animals that were studied, the average islet yield from a pancreas was 485 , with a range from 130 to 873 islets. These data are illustrated in Table 1 .

The number of islets isolated from an individual pancreas was dependent on the concentration of the collagenase solution injected $(P<0.005)$ and on whether it was injected directly into the ductal system. The maximal number of islets was extracted with a collagenase solution of $3.5 \mathrm{mg} / \mathrm{ml}$. Higher and lower concentrations gave lower yields as did parenchymal injection (Table 1).

\section{Microscopy}

With dark-field illumination, islets were spherical in shape and milky white in appearance. With phase-contrast microscopy, islets were identified as brown spheres that were homogeneous in texture. Islet diameters ranged from 90 to $400 \mu \mathrm{m}$. Examination of the islet isolates with phase-contrast provided a qualitative impression that the preparations contained $85-90 \%$ islets, with the remaining structures being acinar cells and ducts.

\section{In Vitro Perifusion of Isolated Islets}

Insulin secretion on exposure to a glucose concentration of $0.3 \mathrm{mg} / \mathrm{dl}$ decreased from $2.38 \pm 0.76(n=6)$ until a baseline secretory rate of 0.50 microunits/islet/min was established. On subsequent exposure to a glucose concentration of $3.0 \mathrm{mg} / \mathrm{dl}$, a biphasic pattern of stimulated insulin release was demonstrated (see Fig. 1). There was an early first phase release of insulin to a level of 2.63 $\pm 1.26(n=4) \mathrm{microunits}$ insulin/islet $/ \mathrm{min}$ at $38 \mathrm{~min}$ and a second peak of $3.28 \pm 0.61$ $(n=4)$ at $75 \mathrm{~min}$. Insulin secretion after 75 min appeared to be turned off by reexposure to a low glucose concentration.

\section{DISCUSSION}

Individual islets are more desirable for use as islet allografts and for in vitro study of islet function than dispersed pancreatic fragments. In this study, we have reported a technique for the isolation of large numbers of viable islets from the pancreas of the Syrian golden hamster. The use of the Syrian golden hamster has increased in popularity over the past two decades because of the rodent's response to stimuli which can alter the growth and development of the pancreas [12].

The standard collagenase digestion technique for the separation and isolation of islets of Langerhans involves the initial distension of the pancreas by a ductal injection of physiological saline, mincing of tissue, and incubation of the pancreatic fragments in a collagenase solution. The final step is the centrifugation of the dispersed tissue on a Ficoll density gradient. With this technique, less than 200 islets per gland have been retrieved [13, 14]. As a consequence of this situation, up to six donors are required to provide sufficient islet mass to reverse diabetes in one rodent recipient $[13,14]$.

Ductal distension and disruption with saline exposes interlobular planes to the indiscriminant digestion by collagenase of both acinar and islet components during the incubation period [15]. Furthermore, mincing of the pancreas causes a $50 \%$ loss of tissue insulin content [16]. Finally, incubation of the 
chopped pancreas in collagenase destroys more than $90 \%$ of the islets as well as acinar tissue. Until quite recently, the isolation of pancreatic islets has been facilitated by the use of density gradient centrifugation. Ficoll is most commonly used to establish a density gradient, but has a number of undesirable properties $[17,18]$. First, it may need to be dialyzed to remove factors which interfere with subsequent B cell function [1]. Second, at densities required for islet separation, Ficoll is hypertonic and has a high viscosity with toxic osmotic properties [19]. The procedure we have described eliminates the potential islet toxicity of Ficoll, as well as the time required to prepare it (dialysis, lyophilization, creation of the gradient).

Our procedure for the isolation of functional islets from the pancreas of the Syrian golden hamster involves the ductal injection of a collagenase solution followed by a period of stationary incubation to allow for digestion of exocrine tissue. The volume and concentration of the digestion medium which is injected, as well as the duration of stationary digestion, may vary with the lot and type of collagenase purchased. While our method permits the isolation of large numbers of islets, some acinar contamination is unavoidable.

Islet yield is most dependent on the success of the collagenase digestion, which is the crucial step in any islet isolation procedure.
During our early experience with this technique, it became apparent that a somewhat lengthened digestion time resulted in an islet preparation with a minimum of other cell types, but at a cost of overall yield and function. The final step of islet isolation is accomplished by differential steel mesh filtration. The size of the islets of Langerhans in the pancreas of the adult hamster has previously been reported $[7,20]$. The diameter of most islets is greater than $140 \mu \mathrm{m}$, but there is a smaller number of islets that are $90-120 \mu \mathrm{m}$. Consideration of islet size was instrumental in choosing steel mesh filters which would provide optimal endocrine separation with minimal contamination from acinar debris.

The isolation of more than 500 islets, on average, from the small pancreas of the hamster represents an increase of more than 350 islets (i.e., 130\%) over that reported previously obtained from the hamster pancreas [7]. Indeed, the number of islets isolated per pancreas in this study represents a significant improvement over the number of islets obtained from the rat pancreas using standard techniques-a gland which has twice the mass of the hamster pancreas $[8,13,14]$. Recently intraductal collagenase injection of the rat pancreas, in a manner similar to what we have described in this study, has been reported to yield, on average, over 600 islets per pancreas [14].

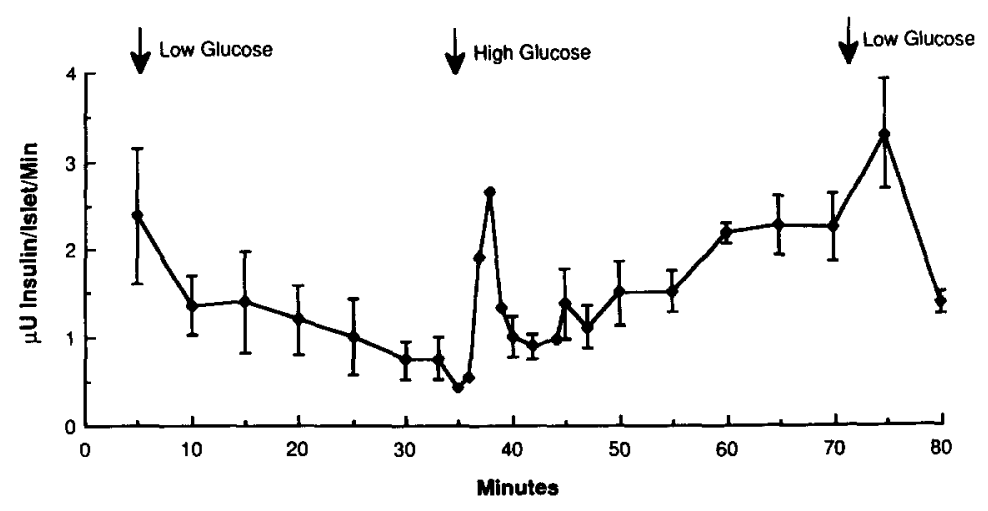

FIG. 1. Biphasic pattern of insulin secretion following stimulation of perifused hamster islets with glucose. The islets studied were isolated from animals $(N=6)$ that were duct injected with $3 \mathrm{ml}$ of collagenase solution $(3.5 \mathrm{mg} / \mathrm{ml}, n=5 ; 4.0 \mathrm{mg} / \mathrm{ml}, n=1)$. 
In vitro perifusion of isolated hamster islets produced a biphasic insulin response to a high glucose concentration, with an early first phase peak representing the release of preformed insulin and a later second phase peak representing the release of newly formed insulin [10]. Furthermore, on reexposure to a low glucose concentration, insulin secretion decreased by $75 \%$. This demonstration of normal physiologic function, coupled with a normal structural appearance, indicates that hamster islets isolated by the collagenase duct injection/steel mesh filtration method are not adversely affected by the isolation process.

We have developed a method for islet preparation whereby the pancreas is selectively digested by injection of the ductal system with a collagenase solution. The common duct is cannulated and collagenase is introduced by slow injection. This system permits improved islet yields with minimal acinar contamination. Morphologic integrity is maintained and islet function, in an in vitro perifusion system, is preserved.

\section{REFERENCES}

1. Sharp, D. W., Kemp, C. B., Knight, M. J., Ballinger, W. F., and Lavy, P. E. The use of Ficoll in the preparation of viable islets of Langerhans from the rat pancreas. Transplantation 16: 686, 1973.

2. Bell, R. H., Sayen, H. J., and Strayer, D. S. Streptozotoxin prevents development of pancreatic cancer in the Syrian hamster. Surg. Forum 33: 437, 1982.

3. Moskalewski, S. Isolation and culture of the islets of Langerhans of the guinea pig. Gen. Comp. Endocrinol. 5: 342, 1965.

4. Lacy, P. E., and Kostianovsky, J. Method for the isolation of intact islets of Langerhans from the rat pancreas. Diabetes 16: 35, 1967.

5. Bowen, K. M., Anders, L., and Lafferty, K. J. Successful allotransplantation of mouse pancreatic islets to nonimmunosuppressed recipients. Diabetes 29(Suppl. 1): 98, 1980.

6. Faustman, D., Hauptfeld, V., Lacy, P., and Davie, J. Prolongation of murine islet allograft survival by pretreatment of islets with antibody directed against Ia determinants. Proc. Natl. Acad. Sci. USA 78: $5156,1981$.

7. Feldman, J. M., and Chapman, B. Preparation of islets of Langerhans from rabbits and hamsters by the collagenase digestion technique. Acta Diabetol. Lat. 12-13: 208, 1975-1976.

8. Gray, D. W. R., McShane, P., Grant, A., and Morris, P. J. A method for isolation of islets of Langerhans from the human pancreas. Diabetes 33: $1055,1984$.

9. Horaguchi, A., and Merrell, R. C. Preparation of viable islet cells from dogs by a new method. Diabetes 30: $455,1981$.

10. Lacy, P. E., Walker, M. M., and Fink, C. J. Perifusion of isolated rat islets in vitro. Participation of the microtubular system in the biphasic release of insulin. Diabetes 21: 987, 1972.

11. Hayashi, M., Floyd, J. C., Pek, S., and Fajans, S. S. Insulin, proinsulin, glucagon and gastrin in pancreatic tumors and in plasma of patients with organic hyperinsulinism. Clin. Endocrinol. Metab. 44: 681, 1977.

12. Homburger, F. The Syrian golden hamster in chemical carcinogenesis research. Prog. Exp. Tumor Res. 10: $137,1968$.

13. Matas, A. J., Sutherland, D. E. R., Steffes, M. W., and Najarian, J. S. Islet transplantation. Surg. Gynecol. Obstet. 145: 757, 1977.

14. Sutton, R., Peters, M., McShane, P., et al. Isolation of rat pancreatic islets by ductal injection of collagenase. Transplantation 42: 689, 1986.

15. Scharp, D. W., Downing, R., Mcrrell, R. C., and Greider, M. Isolating the elusive islet. Diabetes 29: 19-30, 1980.

16. Mirkovitch, V., and Campiche, M. Successful intrasplenic autotransplantation of pancreatic tissue in totally pancreatectomized dogs. Transplantation 21 : 265, 1976.

17. Brunstedt, J. Rapid isolation of functionally intact pancreatic islets from mice and rats by Percoll gradient centrifugation. Diabete Metab. 6: 87, 1980.

18. Lake, S. P., James, R. F. L., Anderson, J., Chamberlain, J., Gardner, S., and Bell, P. R. F. An improved method for rat pancreatic islets. Transplant. Proc. 18: 1817, 1986.

19. Tze, W. J., Wong, F. C., and Tingle, A. J. The use of Hypaque-Ficoll in the isolation of pancreatic islets in rats. Transplantation 22: 201, 1976.

20. Rosenberg, I., Brown, R. A., and Duguid, W. P. Induction of experimental nesidioblastosis: A model to study pancreatic islet cell differentiation and function. Surg. Forum 33: 227, 1982.

21. Scharp, D. W. Isolation and transplantation of islet tissue. World J. Surg. 8: 143, 1984.

22. Sutherland, D. E. R., and Najarian, J. S. Pancreas and islet transplantation. In J. R. Brooks (Ed.), Surgery of the Pancreas. New York: Saunders, 1983. P. 434. 